\title{
Communicative Practices in Their Semantic Orientation on Algorithms of Successful Interactions
}

\author{
Tatiana Leshkevich \\ Southern Federal University \\ Rostov-on-Don, Russia \\ Leshkevicht@mail.ru
}

\begin{abstract}
The article is devoted to the analysis of communicative practices congruent with the socio-cultural type of the social system. The semantic scope of the concept of "communicative practices", their advantages, functions and structural components are discussed. The main purpose of the article is to reveal the threefold significance of communicative practices. They are manifested as the tools for constructing the image of reality, as the ways of reflecting the features of individual consciousness and as the means of shaping intersubjective interactions. The author shows that the fielddependence - field-independence are the basic determinants of communicative style. Communicative practices are analyzed on the basis of an interdisciplinary approach with reference to conceptual ideas by E. Bern, P. Bourdieu, and G. Shchedrovitsky. The focus of attention is shifted to the semantic aspect of the communicative process and basic communication strategies. Particular attention is given to the effects of successful communicative influence and communication patterns accompanying them. The author views manipulation as "the greatest evil" of the 21 st century and offers an analysis of manipulation tactics. In conclusion, the author emphasizes that the basic requirement of the modern age is the ability of individuals and social groups to avoid pitfalls of intercultural interaction, using successful communication patterns.
\end{abstract}

Keywords-communicative practice; communication functions; manipulation; communicative patterns

\section{INTRODUCTION}

Social reality has always been viewed as a field of interactions of communicating individuals. It is penetrated with a variety of communicative practices. Communicative practices are the significant factors that accompany human behavior. They can precede actions, generate actions and derive from specific people's actions. They act as regulators of the life search (quest for life?), relying on rational and non-rational, verbal and extraverbal forms of communication. They contain standards, priorities and orientations of the epoch: civic positions, pragmatic goals, the ideals of justice, love of freedom, humanism, and spirituality [1].

Researchers emphasize that modern communication is congruent with the sociocultural type of the social system in many respects. From the moment of his birth, a person is involved in a network of communicative relations of his environment, culture, epoch. His initial socialization and further development of his personality largely depend on those encounters and interactions that occur throughout his life. Within the framework of communicative practices, selecting and mastering effective norms and values take place, as well as the formation of new preferences, that claim to be generally valid. Therefore, the analysis of the semantic orientation of communicative practices is extremely relevant. Treating convoluted verbal-conceptual constructions and meaningless statements with suspicion, Modernity should address the individual in a language that preserves the national cultural code.

\section{COMMUNICATIVE PRACTICES AS A PROJECTING OF THE RELATION OF HUMAN CONSCIOUSNESS TO REALITY}

Offering the assessment of current events, communicative practices testify to the inclusion of a person in objective reality. Demonstrating a particular cognitive style, they show a person's intelligence level. On the one hand, the term "practice" indicates the embeddedness into "the sociality itself, emphasizes regularity, reproducibility and stereotype of communicative algorithms. On the other hand, communicative practices incorporate motivation, reflect the emotional system, characterize the mental state, reveal the significance of volitional impulses. They overcome the barriers of a purely rational comprehension of the deep layers of human life, relying on intuition, metaphor and association. Thus, communicative practices manifest the relation of human consciousness to reality and promote objectification of a man's inner world. At the same time, communicative practices cannot be constructed on the basis of one's "own will" alone, but they have to be correlated to a huge number of the conditions, rules and restrictions, developed by society.

In this regard, "field-dependence-field-independence" should be identified as the most significant determinants of communicative style. "Field-independence" can be described as the ability to differentiate one's Self, one's own interests and states from external influences and circumstances. It is characterized by the structuredness of self-organization, inflated self-esteem, dismemberment of perception and thinking. On the contrary, "field-dependence" reflects the inadequate differentiation of the Self from the non-Self, blurred self-image, self-depreciation, orientation toward the external environment, global perception and thinking. These 
characteristics determine both communicative style and a person's behavior.

"Listening" being as significant a communicative component as "speaking", communication as a projection of the relation of human consciousness to reality performs the following functions. The first one is a correction function. Having an information component, communication contributes to the adjustment of goals. The second is an expert function of communication. Communicative practices test the situation in a specific way. The third is a reflexive function. Communication process helps to understand the system of individual and social values, expectations and preferences. The fourth is a predictive function. Communication offers clarification or formation of personal and public preferences, taking into account the features of "event space and time". Finally, the fifth function demonstrates the dependence on psycho-mental orientations of communicating subjects, their sympathies and antipathies, self-identification. This makes the identification function. The combination of these functions is a powerful advantage of communicative practices.

\section{METHODOLOGICAL STRATEGY AND THEORETICAL BACKGROUND OF THE STUDY INTO COMMUNICATIVE PRACTICES}

Modern analysis of communicative practices should be carried out in the context of an interdisciplinary approach. If we refer to the socio-humanitarian potential of interdisciplinary research, then the leading role belongs to philosophy, sociology, psychology, linguistics, coupled with education paradigm and cognitive studies. Thus, philosophy shows their dependence on the epoch and socio-cultural conditions of existence. The principle of sociocultural determination and the thesis: "being determines consciousness" is still essential. It should be emphasized that the modern information civilization has made considerable changes, expending communicative practices to new forms of the Internet language. The book with the remarkable title "The Russian Language on the Verge of a Nervous Breakdown" suggests that the language style of a mass Internet user is not constrained by any cultural framework and limitations [2]. This is a kind of communicative revolution made by online communication. This process leads to irreversible communicative shifts, right up to a communicative gap between generations. In general, as studies show, communicative risks are connected with social mechanisms of innovative activity [3].

Sociologists have long recognized the fact that representatives of different social groups use different types of articulation with specific discursive features. This enables us to record incommensurability and at the same time the pronounced "inner sociality" of certain interacting groups. Sociologists seek to establish dependency between communicative practices and the stratum, the status of an individual on "a social prestige staircase". Professional affiliation and specific characteristics of a social group, which the individual belongs to, have a great influence on communication patterns [4]. Thus, the language of a diplomat will differ from the language of a merchant in many ways. The language of an intellectual, keen on book culture, is other than a villager's language. Ethnography shifts the focus of attention on mundane and cultural peculiarities of communication in ethnic areas.

From the point of view of the cognitive approach, human brain processes information in order to comprehend and explore the environment and to determine an adequate behavior strategy. Distributive processing of incoming information is accompanied by mental representations. Linguistics studies the semantic and syntactic transformations of language and speech acts in the context of the historical formation of the language environment. Paralinguistics specializes in the study of semantic information expressed through non-verbal communication: gestures, facial expressions, postures and other non-verbal communication means.Extra-linguistic forms of communication (pauses, laughter, crying) are also significant It is no coincidence that there is an expression the "sacrament of communication", indicating a huge potential of non-verbal communication.

Psychology reveals the dependence of communicative practices on the emotional characteristics and temperament of an individual, analyzes communicative abilities and multiplicity of communicative ways of self-expression [5]. Psychology dwells on both the features of communicative behavior and destabilizing factors with regard to social interactions. According to researchers, communication performs the function of constructing interpersonal space [6]. Psychologists reveal the specificity of open communication in contrast to institutional communication [7] [8]. E. Berne's monograph, which identifies six basic types of communicative behavior, occupies a special place. They are: 1) avoiding communication; 2) spending time together without drawing closer; 3) communication aimed at social selection and useful contacts; 4) communication aimed at obtaining profit; 5) communication as a non-profit intimacy; 6) interpersonal interaction in joint activity [9]. Such a classification enables each person to discover his personal specific way of communication.

Within educational paradigm, the central place is taken by persuasive communication, aimed at achieving the consent of both parties [10]. Argumentation techniques, perseverance, as well as references to authority and coalition provide the effect of persuasive communication [11]. The analysis of such communicative strategies as presentation, manipulation, convention, accompanied by appropriate technologies, is of particular practical importance [12]. However, the process of education also comprises the socalled ritual communications, for example, the style of greetings, farewells, addresses, etc. They do not provide for alternative actions, there is no involvement in meaningful relationships; the choice of action is narrowed by the partners in the ritual [13]. However, it is necessary to emphasize that communication adds a certain semantic orientation to the phenomena of the surrounding reality. The subject alters the situation through communication, changing its semantic form [14]. 
Semantic analysis of the concept "communicative practices" reveals dynamic and subject-activity component, which makes it possible to interpret them as an integral part of sociality itself. This concept enables us to see human life through the lens of people's actions. [15]. By introducing the concept "habitus", P. Bourdieu got quite close to determining the value of practices. According to Bourdieu, "habitus" is a general program or a set of principles, which lie behind various practices and assume their indispensable implementation. It suggests the choice of one or another practice. Hence, it is clear that practices encompass a kind of intuition, explicit and implicit rules [16].

Epistemological characteristics of communicative practices are connected with the application of various cognitive styles, cognitive simplicity-complexity, reflexivity-impulsivity, smoothing, - sharpening being among them [17]. We should also distinguish between different layers of communication: habitual (a pattern given by sociality) and reflexive (associated with a unique personal understanding). The epoch is represented by a variety of different forms of communicative practices, including marginal forms along with legitimate ones. There is a concept of "distorted communication", which is based on a powerful lobby, the lack of publicity and transparency. Its purpose is to conceal diverging interests.

Thus, the advantages of an interdisciplinary approach to the study of intercultural communication are as follows. The first one consists in the fact that, integrating the data of modern sciences, it explains specificity of diverse communicative technologies. Secondly, overcoming disciplinary boundaries, an interdisciplinary approach shows the ways of communicating adaptation methods to the next generation, sharing experience and the meanings of social actions.

\section{STRUCTURE OF COMMUNICATION AND THE EFFECTS OF COMMUNICATIVE INFLUENCE}

Figuratively speaking, communication contributes to defining moves "in a chess game of human life." Its components are the author, his discourse and an actual or a potential partner. The process of direct communicative interaction, as well as a message reflecting the content of communication, are of fundamental importance. Communication parties claim that their statements are either true, or correct, or personally meaningful. Communication processes are dependent on many "anonymous contexts", but every person seeks to build connections with others, attaching to it the intentions of one's own subjective influence.

Within a formalist approach to the communication structure, the following components are distinguished: a communicant (information sender) - message - a recipient (receiver of information). The effects of communication process largely depend on whether the information is sent to individual persons (axial communication), or spread to a great number of recipients (retinal communication - from the Latin network).
By shifting the focus of attention to the semantic aspect of communicative process, the following components acquire significance. They are: a person who shapes meanings; the subjective world of experience; the world of possible expectations; the external objective world; a set of conventional norms and role relations. The existence of each person is plunged into this multilayered process, which, however, may remain non-conscious. At the same time, a reflexive analysis of communicative influence makes it possible to register the stages of pre-understanding, interpretation and correction of understanding. Openness and the focus on the other party's response, coupled with the need for new meanings, are the cornerstones of semantic attraction. Communication processes can take the shape of a monologue, dialogue or polylogue, but it is dialogue practices that appear to be the most relevant. An eloquent expression "hear me out" testifies to the need for a successful dialogue and mutual understanding. The semantics of a new concept "negotiators" pursues the same goal. It refers to those undertaking the mission of having a constructive dialogue.

From the perspective of a logical-conceptual approach, the effects of communicative impact are four-fold: one side may dominate and absorb the other; the two sides find common ground and are inclined to reach consensus; the communicating parties establish a new platform for discussion; communicative practice is accompanied by the suppression of communicating parties and leads to a communicative failure.

Within the context of contemporary intercultural communication, communication strategies aimed at developing successful impact algorithms are of particular interest. The effect of successful influence is connected with the so-called "communicative keys" associated with the context of communication, adaptation to the situation, practicability and functionality [18]. If we dwell on the effect of successful communication, it is necessary to consider a number of patterns aimed at its actualization. The pattern of communication predisposition is one of them. The pattern of communication congruence, which indicates the readiness for perspective coordination of communicative actions, is another significant pattern. The pattern of communicative complementarity presupposes the desire to complement and continue what has been initiated by the partner. The pattern of communicative equivalence reflects the desire to keep pace with the partner and reciprocate. The most widespread is the pattern connected with the pursuance of self-affirmation in the process of communication.

It should be emphasized that manipulative techniques are often used to achieve a successful communication impact. Scholars and public figures view manipulation as the greatest evil of the XXI century. According a well-known Russian methodologist G. Shchedrovitsky, manipulation coexists with such types of communicative strategies as presentation and convention [19]. Using hidden mechanisms of influence, manipulation presupposes transmitting an external aim to a chosen subject. Within this context, the mindset of an agent lacks understanding of his own actions, shaping incompetence. Researchers distinguish between two main 
goals of manipulation, which are aimed at either motivating or preventing an action. The effect of achieving this goal is provided by the means of specific tactics of manipulative communication.

- Six tactics of manipulative communication are the most widespread.

- Persuasion, request ("I am asking you to do this, because ...").

- Retreat, regress ("I'll be crying unless you do this...").

- Coercion ("I demand ...").

- Charm ("You are a wiz at that ...").

- Humiliation ("I will be kneeling unless you do this...").

- Silence (until the recipient makes a concession) [20] [21].

In practice, tactics of charm are more often used when the interlocutor is induced to act, while tactics of silence and coercion are aimed at stopping an undesirable action. However, in addition to manipulation, interactive intersubject communications can manifest themselves in the form of confrontation, compromise, avoiding discussion or neutrality. All the above-mentioned are accompanied by certain means of influence, advice and recommendations of the expert community.

\section{CONCLUSION}

Our study into the problem leads to the following conclusions. Communicative domain appears to be a very noisy sphere, filled with the emissions of different-quality energies, derivatives of social entropy. The process of communication consists in the circulation of traditional meanings as well as the formation of new one, including both archetypes and stereotypes.

The circulation of semantic, emotional, verbal and other types of information shapes a broad communicative field, the boundaries of which are not limited to the present alone, but extend to both the past and the future. At the same time, communicative practices contain the ability to thematize and "work through" contradictory situations as well as to penetrate into the domain of needs and motivations, inner motives and critical assessment. Thus, it can be concluded that communicative practices provide adaptation, act as a specific compensatory mechanism and, eventually, define the vector of social action. Intercultural communication contributes to the formation of a special type of determination - determination by the future.

Post-informational epoch brings about modifications to inter-cultural communicative practices. Being intelligent is no longer enough. Being technologically-minded and capable of functioning in the context of a global information civilization seems to be a fundamental requirement. Information content has become the primary resource of modern civilization development [22]. Under these conditions, successful inter-cultural communication, the ability of the main social groups to communicate and overcome risky situations is the leading priority of modern Russia.

\section{REFERENCES}

[1] O. V. Chistyakova, Relationship of Self and Other in Cultural and Religious Communications // Proceedings of the International Conference on Education, Language, Art and Inter-Cultural Communication. Xiamen, 2016 (ICELAIC 2016). Advances in Social Science, Education and Humanities Research, Volume 40. Paris: Atlantis Press, 2017. ISBN (on-line): 978-94-6252-292-3 - pp. 660664

[2] M. A. Krongauz, "The Russian Language on the Verge of a Nervous Breakdown," Moscow: Languages of Slavic Cultures, 2008.

[3] I. T. Kasavin, "Social epistemology. Fundamental and applied problems ", M: Alfa-M, 2013.

[4] A. P. Panfilova, "Business Communication in Professional Activity", St. Petersburg: The Union, 2001.

[5] A. K. Bolotova, Yu. M. Zhukov, "Psychology of Communications," M: Publishing House of Higher School, 2015

[6] E. L. Dotsenko, "The Psychology of Manipulation: Phenomena, Mechanisms and Protection", St. Petersburg: Speech, 2003.

[7] A. A Bodalev, "Psychology of Communication", M: Publishing Institute of Practical Psychology-Voronezh, 1996.

[8] R. M. Arkin, "Self-presentation styles", Impression Management Theory and Social Psychological Research / J. T. Tedeschi (ed.), N.Y. Academic Press, 1981.

[9] E. Bern, "Games played by people." People who play games', M: Progress, 1988

[10] G. M Andreeva, "Social psychology", M: Aspect-Press, 2003.

[11] E. V Sidorenko, "Training of communicative competence in business interaction", SPb: Speech, 2002.

[12] G.P. Shchedrovitsky, "Philosophy. The science. Methodology ", M Shk. Cult of Politics, 1997.

[13] J.C. Krizhanskaya, VP Tretyakov, "Grammar of communication", St Petersburg: Meaning, 2005.

[14] T.G. Leshkevich, "Language as a sign reality; horizons and metamorphoses ", Problems of language in the global world, M: Publishing house Prospekt, 2016, p.104.

[15] B.G. Gutner, "Post-Classical Study: the Communicative Character of Practitioners", Post-Classical Practices: The Experience of Conceptualization, St. Petersburg: Mir, 2012, p. 137.

[16] P. Bourdieu, "Structures, habitus, practices", Modern social theory: Bourdieu, Giddens, Habermas, Novosibirsk: NSU Press, 1995. p. 44.

[17] V.V. Selivanov, "Cognitive style", / Encyclopedia of epistemology and philosophy of science, M: Canon, 2009, p. 36

[18] T.G. Leshkevich, "Communicative nature of social and humanitarian knowledge" / Philosophy of Science, Rostov-on-Don, Phoenix, 2006. pp. 305-306.

[19] G.P. Shchedrovitsky, "Philosophy. The science. Methodology ", M Shk. Cult of Politics, 1997.

[20] R. Chaldini, "Psychology of Influence". St. Petersburg: Piter, 2000

[21] F. Zimbardo, M. Leippe, "Social influence", St. Petersburg: Piter, 2000. p. 575

[22] T.G. Leshkevich, "The role of subjectivity in the formation of a global way of thinking," Dialogue of Cultures: Social, Political and Value Aspects, M.: Canon, 2015. p. 575. 